\title{
Response to 'Comment on 'The potential contribution of tumour-related factors to the development of FOLFOX-induced sinusoidal obstruction syndrome"
}

\author{
S M Robinson *,1,2, D A Mann ${ }^{2}$, D M Manas ${ }^{1}$, F Oakley $^{2}, \mathrm{~J} \mathrm{Mann}^{2}$ and S A White ${ }^{1,2}$ \\ ${ }^{1}$ Department of HPB Surgery, Freeman Hospital, Newcastle upon Tyne, NE7 7DN, UK and ${ }^{2}$ Institute of Cellular Medicine, Newcastle University, \\ Newcastle upon Tyne, NE2 4HH, UK
}

Sir,

We read with interest the observations of Lentschener et al., who have attempted unsuccessfully to replicate our murine model of FOLFOX-induced sinusoidal obstruction syndrome (SOS) (Robinson et al, 2013a, b). While the drug treatment regimen and experimental conditions used in these experiments were broadly similar to those used by us, we do note some key important differences.

During our initial attempts to develop a model of FOLFOX-induced SOS, we similarly found that animals maintained on standard chow diets did not develop the histological features of SOS - a finding described in our original description of this model (Robinson et al, 2013a). We observed that switching to a purified diet (D01060501, Research Diets Inc, New Brunswick, NJ, USA) but maintaining an otherwise identical experimental protocol did lead to the development of histological features of SOS. We have previously hypothesised (although not proven) that this may be attributable to the presence of phytoestrogens in standard animal diets, which have a protective effect on the development of liver injury (Ascencio et al, 2004; McCarty et al, 2009). While the diet utilised by Lentschener et al. does contain reduced phytoestrogens as compared with standard chow diets (Global Rodent diet-2016, Harlan Laboratories, Madison, NJ, USA), these are still present. Of course it may be that other constituents in the diet are having a role, but we have not explored this.

It would be also interesting to know which substrain of C57BL/6 mouse was utilised by Lentschener et al. In our experiments, we utilised the $\mathrm{C} 57 \mathrm{BL} / 6 \mathrm{~J}$ substrain that differs from the C57BL/6N substrain by the deletion of exons $7-11$ of the nicotinamide nucleotide transhydrogenase $(\mathrm{Nnt})$ gene that has key roles in the mitochondrial response to oxidative stress (Mekada et al, 2009; Simon et al, 2013). As we have previously reported, oxidative stress appears to have a key role in the development of SOS, and therefore the choice of C57BL/6 substrain may be particularly important in this context, although we haven't specifically explored this.

The final observation to make is that all drugs in our description of the model were obtained from Sigma-Aldrich (Dorset, UK), whereas those used by Lentschener et al, were from different sources. Whether these different preparations have the same pharmacological characteristics in vivo is not known.

While we do believe that FOLFOX-induced SOS can be modelled in mice, we acknowledge that the work of Lentschener et al, does highlight how subtle differences in the experimental protocol can have a significant impact on the reproducibility of this model.

\section{CONFLICT OF INTEREST}

The authors declare no conflict of interest.

\section{REFERENCES}

Ascencio C, Torres N, Isoard-Acosta F, Gomez-Perez FJ, Hernandez-Pando R, Tovar AR. (2004) Soy protein affects serum insulin and hepatic SREBP-1 mRNA and reduces fatty liver in rats. J Nutr 134(3): 522-529.

McCarty MF, Barroso-Aranda J, Contreras F. (2009) Genistein and phycocyanobilin may prevent hepatic fibrosis by suppressing proliferation and activation of hepatic stellate cells. Med Hypotheses 72(3): 330-332.

Mekada K, Abe K, Murakami A, Nakamura S, Nakata H, Moriwaki K, Obata Y, Yoshiki A. (2009) Genetic differences among C57BL/6 substrains. Exp Anim 58(2): 141-149.

Robinson SM, Mann J, Vasilaki A, Mathers J, Burt AD, Oakley F, White SA, Mann DA. (2013a) Pathogenesis of FOLFOX induced sinusoidal obstruction syndrome in a murine chemotherapy model. J Hepatol 59(2): 318-326.

Robinson SM, Mann DA, Manas DM, Oakley F, Mann J, White SA. (2013b) The potential contribution of tumour-related factors to the development of FOLFOX-induced sinusoidal obstruction syndrome. Br J Cancer 109: 2396-2403.

Simon MM, Greenaway S, White JK, Fuchs H, Gailus-Durner V, Wells S, Sorg T, Wong K, Bedu E, Cartwright EJ, Dacquin R, Djebali S, Estabel J, Graw J, Ingham NJ, Jackson IJ, Lengeling A, Mandillo S, Marvel J, Meziane H, Preitner F, Puk O, Roux M, Adams DJ, Atkins S, Ayadi A, Becker L, Blake A, Brooker D, Cater H, Champy MF, Combe R, Danecek P, di Fenza A, Gates H, Gerdin AK, Golini E, Hancock JM, Hans W, Hölter SM, Hough T, Jurdic P, Keane TM, Morgan H, Müller W, Neff F, Nicholson G, Pasche B, Roberson LA, Rozman J, Sanderson M, Santos L, Selloum M, Shannon C, Southwell A, TocchiniValentini GP, Vancollie VE, Westerberg H, Wurst W, Zi M, Yalcin B, RamirezSolis R, Steel KP, Mallon AM, de Angelis MH, Herault Y, Brown SD (2013) A comparative phenotypic and genomic analysis of C57BL/6J and C57BL/6N mouse strains. Genome Biol 14(7): R82

This work is published under the BJC's standard license to publish agreement. After 12 months the license terms will change to a Creative Commons AttributionNonCommercial-Share Alike 4.0 Unported License. 